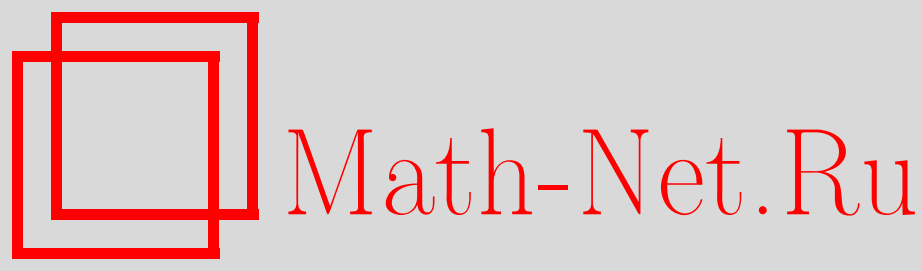

В. М. Журавлев, Многомерные нелинейные волновые уравнения с многозначными решениями, ТМФ, 2013, том 174, номер 2, 272-284

DOI: https://doi.org/10.4213/tmf8391

Использование Общероссийского математического портала Math-Net.Ru подразумевает, что вы прочитали и согласны с пользовательским соглашением http://www. mathnet.ru/rus/agreement

Параметры загрузки:

IP : 54.198 .55 .26

26 апреля 2023 г., 08:27:51

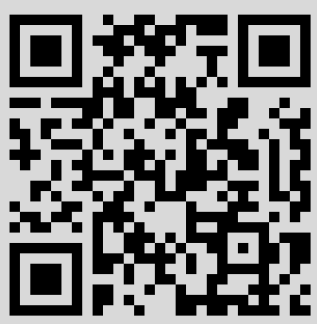




\title{
МНОГОМЕРНЫЕ НЕЛИНЕЙНЫЕ ВОЛНОВЫЕ УРАВНЕНИЯ С МНОГОЗНАЧНЫМИ РЕШЕНИЯМИ
}

\begin{abstract}
Изложена теория опрокидывающихся волн в нелинейных системах, описание динамики и пространственной структуры которых строится на базе многомерных нелинейных гиперболических волновых уравнений. Найдена общая связь систем квазилинейных уравнений первого порядка с нелинейными гиперболическими уравнениями более высокого порядка, которые описывают, в частности, электромагнитные волны в среде с нелинейной поляризацией произвольного вида. В рамках этого подхода построены точные многозначные решения уравнений этого типа. Исследованы пространственная структура этих решений и их динамика. Результаты переносятся на широкий класс многомерных уравнений, таких как уравнения Даламбера, нелинейные уравнения Клейна-Гордона и нелинейные телеграфные уравнения.
\end{abstract}

Ключевые слова: точные решения многомерных гиперболических уравнений, опрокидывающиеся волны, многозначные решения, электромагнитные волны в среде с нелинейной поляризацией.

DOI: $10.4213 / \operatorname{tmf} 8391$

\section{1. ВВЕДЕНИЕ}

Многозначные функции являются одним из основных атрибутов теории квазилинейных уравнений первого порядка, которые возникают в различных прикладных задачах гидродинамического типа [1]-[3]. В таких задачах многозначные решения заменяются на однозначные разрывные решения - ударные или опрокидывающиеся волны. Однако известно сравнительно небольшое число многомерных уравнений порядка выше первого, для которых многозначные решения построены в явном виде. K уравнениям такого типа относятся, например, некоторые уравнения теории солитонов, описанные в монографии [4]. Интересный, но, по всей видимости, в значительной степени забытый результат содержится в книге [5], где описаны многозначные решения и ударные волны уравнений Максвелла для определенных типов нелинейных сред и систем. В нелинейной оптике такие решения найдены в работе [6]. Важным и уже ставшим классическим результатом является утверждение о наличии многозначных решений уравнения Даламбера в размерности $3+1$, что

*Ульяновский государственный университет, Ульяновск, Россия.

E-mail: zhvictorm@gmail.com 
было использовано в теории твисторов и точных решений в теории гравитации в общей теории относительности [7], [8]. В работе [9] был найден класс многозначных решений уравнения Лиувилля в размерности $3+1$, который был распространен на нелинейное уравнение теплопроводности в размерности $4+1$. В работе [10] было показано, что для гиперболического уравнения второго порядка, описывающего распространение электромагнитных волн в диэлектриках с произвольного вида поляризуемостью среды в случае отсутствия дисперсии, существует класс точных решений, которые представляют собой решения квазилинейного уравнения первого порядка с подходящего вида локальной фазовой скоростью, зависящей от напряженности волны. Этот же результат справедлив для уравнений, описывающих нелинейные акустические волны. Из этих примеров видно, что уравнения с многозначными решениями встречаются в различных разделах физики и часто играют в них очень важную роль. Поэтому поиск общих классов решений уравнений, которые содержат многозначные решения, может оказаться важным фактором в понимании общего устройства множества решений нелинейных гиперболических уравнений, широко используемых в практических задачах.

В настоящей работе, основываясь на результате работы [10], строится новый класс точных многозначных вещественных решений целого ряда многомерных (в размерности $n+1)$ нелинейных уравнений второго порядка, в том числе уравнений распространения электромагнитных волн в диэлектриках с произвольной поляризуемостью и акустических волн в газах. Источником новых решений являются многомерные квазилинейные уравнения первого порядка. Отметим, что векторные уравнения такого типа для размерности координатного пространства $n=1$ (в размерности $1+1$ ) рассматривались в рамках обобщенного метода годографа [11] и для размерности $n=2$ (в размерности $2+1)$ - в рамках метода гидродинамических редукций [12], [13]. В этих работах исследовались условия интегрируемости таких систем и анализировались их общие свойства. В настоящей работе в качестве источника решений рассматриваются квазилинейные уравнения относительно одной скалярной неизвестной функции, но в размерности координатного пространства $n$ (в размерности $n+1)$. Интегрируемость таких систем устанавливается путем явного указания общего интеграла движения. Результаты построения решений для уравнений второго порядка специального вида распространяются в настоящей работе на уравнения типа уравнения Клейна-Гордона с произвольной конечной размерностью координатного пространства, а также на нелинейные уравнения телеграфного типа той же размерности $n+1$. В настоящей работе рассматриваются уравнения с вещественными коэффициентами и их вещественные решения.

\section{2. СИСТЕМЫ КВАЗИЛИНЕЙНЫХ УРАВНЕНИЙ И МНОГОМЕРНЫЕ НЕЛИНЕЙНЫЕ ГИПЕРБОЛИЧЕСКИЕ УРАВНЕНИЯ}

Рассмотрим систему квазилинейных уравнений следующего вида:

$$
E_{, \alpha}=\frac{\partial E}{\partial x_{\alpha}}=A_{\alpha}(E) E_{t}, \quad \alpha=1, \ldots, n,
$$

где $\mathbf{A}(E)=\left(A_{1}(E), \ldots, A_{n}(E)\right)$ - вектор-функция, зависящая от неизвестной функции $E=E(\mathbf{x}, t), \mathbf{x}=\left(x_{1}, \ldots, x_{n}\right)$, и $t$. 
Решением этой системы является функция $E=E(\mathbf{x}, t)$, удовлетворяющая алгебраическому уравнению

$$
H\left(E, t+A_{1}(E) x_{1}+\cdots+A_{n}(E) x_{n}\right)=0
$$

где $H(\xi, \eta)$ - произвольная дифференцируемая функция двух аргументов $\xi=E$ и $\eta=t+(\mathbf{A}, \mathbf{x})$, где $(\mathbf{A}(E), \mathbf{x})=A_{1}(E) x_{1}+\cdots+A_{n}(E) x_{n}$. Это устанавливается последовательным дифференцированием (2) по переменным $x_{1}, \ldots, x_{n}, t$ :

$$
\begin{aligned}
E_{, \alpha} H_{\xi}+\left(A_{\alpha}+\left(\mathbf{A}^{\prime}(E), \mathbf{x}\right) E_{, \alpha}\right) H_{\eta} & =0, \quad \alpha=1, \ldots, n, \\
E_{, t} H_{\xi}+\left(1+\left(\mathbf{A}^{\prime}(E), \mathbf{x}\right) E_{, t}\right) H_{\eta} & =0 .
\end{aligned}
$$

Исключая из этой системы уравнений производные функции $H(\xi, \eta)$, получаем систему уравнений (1).

Из системы уравнений (1) в результате дифференцирования и повторного использования ее самой находим важное следствие:

$$
\Delta E=\frac{\partial}{\partial t}\left(|\mathbf{A}|^{2} E_{, t}\right)
$$

где

$$
|\mathbf{A}(E)|^{2}=\sum_{\alpha=1}^{n} A_{\alpha}^{2}(E) .
$$

Введем обозначение

$$
\nabla_{|\mathbf{A}|}=\Delta-\frac{\partial}{\partial t}\left(|\mathbf{A}(E)|^{2} \frac{\partial}{\partial t}\right),
$$

так что уравнение (4) можно записать в следующем виде:

$$
\diamond_{|\mathbf{A}|} E=0
$$

Важным свойством уравнения (4), вне зависимости от его интерпретации и типа нелинейности, является инвариантность его формы относительно преобразований вращений вектор-функции $\mathbf{A}(E)$ в координатном пространстве $\mathbb{R}^{n}$. Все такие преобразования оставляют инвариантной длину вектора $\mathbf{A}(E)$ в $\mathbb{R}^{n}$. Введем обозначение $R(E)=|\mathbf{A}(E)|=\sqrt{A_{1}^{2}(E)+\cdots+A_{n}^{2}(E)}$. Тогда вектор-функцию $\mathbf{A}(E)$ можно представить в следующем общем виде:

$$
\mathbf{A}=R(E) \mathbf{n}(E),
$$

где $\mathbf{n}(E)$ - произвольный единичный вектор в пространстве $\mathbb{R}^{n}$, зависящий от значения функции $E$. Следовательно, компоненты вектора $\mathbf{n}(E)$ содержат $n-1$ произвольных функций, которые представляют собой угловые координаты на единичной сфере $\mathcal{S}^{n-1}$, зависящие от $E$.

Инвариантность формы уравнения (4) относительно выбора функциональной зависимости единичного вектора $\mathbf{n}(E)$ от $E$ означает, что решение уравнения (2) при любом допустимом выборе $H(\xi, \eta)$ и $\mathbf{n}(E)$ является решением уравнения (4). При этом следует отметить, что произвольный выбор функции $H(\xi, \eta)$ в силу (2) допускает в решениях системы (1) произвол, ограниченный функцией лишь одного аргумента. 
УтВЕРЖДЕНИЕ 1. Каждое решение системы квазилинейных уравнений (1) для заданной бункции $R(E)=|\mathbf{A}(E)|$ и произвольной вектор- функции $\mathbf{n}(E),|\mathbf{n}|^{2}=1$, является решением уравнения (4). Обратное утверждение неверно.

УТВЕРЖДЕнИЕ 2. Каждое решение системы квазилинейных уравнений (1) для заданной функции $R(E)=|\mathbf{A}(E)|$ и произвольной вектор-функции $\mathbf{n}(E),|\mathbf{n}|^{2}=1$, является решением уравнения эйконала

$$
\sum_{\alpha=1}^{n}\left(E_{, \alpha}\right)^{2}=|\mathbf{A}(E)|^{2}\left(E_{, t}\right)^{2} .
$$

Это утверждение является прямым следствием системы (1).

Еще одно следствие можно получить из системы (1) с помощью дифференцирования ее по $t$ и комбинирования с самой этой системой уравнений. Оно имеет вид уравнения Эйлера.

УтВЕРЖДЕНИЕ 3. Вектор-функиия $\mathbf{U}=\left\{U^{1}, \ldots, U^{n}\right\}$ с компонентами

$$
U^{\alpha}=-\frac{1}{|\mathbf{A}|^{2}} A^{\alpha}(E), \quad \alpha=1, \ldots, n,
$$

и функиия $\rho=|\mathbf{A}|^{2} E_{t}$ удовлетворяют системе однородных уравнений Эйлера

$$
\begin{aligned}
& \frac{\partial U^{\alpha}}{\partial t}+U^{\beta} \frac{\partial U^{\alpha}}{\partial x^{\beta}}=0, \quad \alpha=1, \ldots, n, \\
& \frac{\partial \rho}{\partial t}+\frac{\partial}{\partial x^{\alpha}}\left(\rho U^{\alpha}\right)=0
\end{aligned}
$$

для потока идеальной жидкости со скоростъю $\mathbf{U}$ и плотностъю $\rho$.

Справедливость первого уравнения устанавливается прямым дифференцированием вектор-функции $\mathbf{U}$ и последующим использованием исходной системы уравнений (1). Второе уравнение эквивалентно уравнению (4) при использовании определения для функции $\rho$ и $\mathbf{U}$.

\section{3. ФИЗИЧЕСКАЯ ИНТЕРПРЕТАЦИЯ УРАВНЕНИЙ}

Уравнение (4) преобразуется к уравнению плоскополяризованных электромагнитных волн в среде с нелинейной поляризацией $P(E)$, связанной с вектор-функцией $\mathbf{A}(E)$ соотношением

$$
P^{\prime}(E)=\frac{c^{2}}{4 \pi}|\mathbf{A}(E)|^{2}-1
$$

В этом случае уравнение (4) имеет точный вид уравнения Максвелла для электромагнитных волн в среде с нелинейной поляризацией $P(E)$ :

$$
c^{2} \Delta E-E_{, t t}=4 \pi \frac{\partial^{2}}{\partial t^{2}} P(E) .
$$

Это уравнение соответствует отсутствию дисперсии в среде, что определяет специфически условия его применения в электродинамике. В частности, такие условия описаны в монографии [5]. 
Другая физическая интерпретация уравнения соответствует звуковым волнам в идеальном газе. Уравнения акустических волн в такой среде имеют вид

$$
\begin{aligned}
\frac{\partial}{\partial t} \rho u^{\alpha}+\frac{\partial}{\partial x^{\beta}} \rho u^{\alpha} u^{\beta} & =-\frac{\partial P}{\partial x^{\alpha}}, \\
\frac{\partial}{\partial t} \rho+\frac{\partial}{\partial x^{\beta}} \rho u^{\beta} & =0 .
\end{aligned}
$$

Первое векторное уравнение этой системы представляет собой уравнение Эйлера для скорости потока $u^{\alpha}$, а последнее - уравнение неразрывности. Для адиабатических звуковых волн в газе давление $P$ является заданной функцией плотности газа $\rho$. Вычисляя дивергенцию первого векторного уравнения этой системы, приходим к уравнению для $\rho$ :

$$
\rho_{t t}=\Delta P(\rho)+\frac{\partial^{2}}{\partial x^{\alpha} \partial x^{\alpha}}\left(\rho u^{\alpha} u^{\beta}\right)
$$

Пренебрегая последним слагаемым в правой части (инерционной нелинейностью), получаем уравнение для давления в среде в форме

$$
\Delta P=\frac{\partial^{2}}{\partial t^{2}} \rho(P)
$$

В этом случае $\rho^{\prime}(P)=|\mathbf{A}(P)|^{2}$.

\section{4. ОБЩИЕ СВОЙСТВА РЕШЕНИЙ СИСТЕМЫ КВАЗИЛИНЕЙНЫХ УРАВНЕНИЙ}

Решения уравнения (4), совпадающие с решениями системы квазилинейных уравнений (1), обладают особыми свойствами, которые выделяют их из всех других типов возможных решений этого уравнения. Поскольку векторное поле $\mathbf{A}=\mathbf{A}(E(\mathbf{x}, t))$ в заданный момент времени $t$ зависит только от значения функции $E$, оно постоянно на изоповерхностях функции $E:\left.\mathbf{A}\right|_{E=\text { const }}=$ const. Однако согласно уравнениям (1) в каждой точке пространства поле $\mathbf{A}$ коллинеарно полю $\nabla E$, которое всюду ортогонально гиперповерхности $E=E_{0}(t)$. Отсюда следует, что изоповерхностями функции $E$ являются гиперплоскости, поскольку в любой точке этой гиперповерхности нормаль к ней имеет одно и то же направление, зависящее только от $E$. Как уже отмечалось выше, форма уравнения (4) не зависит от направления поля $\mathbf{A}$ в каждой точке, поэтому каждое решение системы (1), совпадающее с одним из решений уравнения (4) при заданной функции $R(E)=|\mathbf{A}|$, будет определяться вектор-функцией $\mathbf{n}(E)$. Точнее это можно выразить в форме следующего утверждения.

УтвеРЖДЕНИЕ 4. Решение $E(\mathbf{x}, t)$ системы (1) при заданной функции $R(E)=$ $|\mathbf{A}(E)|$ определяется однозначно парой функциональных параметров $\left\{\mathcal{C}_{n}, E(s, t)\right\}$, где $\mathcal{C}_{n}$ является не зависящей от времени однопараметрической кривой, называемой далее базовой, которая задается уравнениями

$$
\frac{d x^{\alpha}}{d s}=n^{\alpha}(s), \quad A^{\alpha}(E(s))=R(s) n^{\alpha}(s),
$$


в координатном пространстве $\mathbb{R}^{n}$, а функция $E(s, t)=E(\mathbf{x}(s), t)$ является решением уравнения Хопфа:

$$
\frac{\partial E}{\partial s}=R(E) \frac{\partial E}{\partial t}
$$

ДокАЗАТЕЛЬство. Поскольку выше было доказано, что изоповерхностями функции $E(\mathbf{x}, t)$ являются гиперплоскости, которым ортогональны поля $\mathbf{A}=R \mathbf{n}$ и $\mathbf{n}$, первую часть утверждения можно считать доказанной. Пусть $\mathcal{C}_{n}-$ кривая, заданная уравнениями (9). Тогда, используя систему уравнений (1), имеем

$$
\frac{\partial E}{\partial s}=\sum_{\alpha=1}^{n} \frac{\partial E}{\partial x^{\alpha}} \frac{\partial x^{\alpha}}{\partial s}=\sum_{\alpha=1}^{n} R(E) E_{t} n^{\alpha}(E(s, t)) n^{\alpha}(E(s, t))=R(E) E_{t} .
$$

Последнее равенство вытекает из условия $|\mathbf{n}|^{2}=\sum_{\alpha=1}^{n} n^{\alpha} n^{\alpha}=1$. Отсюда сразу следует (10). Заметим, что решение $E(s, t)$ уравнения Хопфа находится как решение уравнения

$$
H(E, t+R(E) s)=0
$$

где $H(\xi, \eta)$ - та же функция, что и в решении уравнения (2), это соответствует выбору параметра $s$ по формуле

$$
\sum_{\alpha=1}^{n} n^{\alpha}(E(s, t)) x^{\alpha}(s)=s .
$$

В результате решение уравнения (2) переходит в решение уравнения (12).

Такие решения в дальнейшем мы будем называть ривертонами (от английского "river" - река). Как следует из приведенного утверждения, ривертоны представляют собой бегущие плоские волны, направление распространения которых в каждой точке пространства не меняется со временем и определяется касательной к кривой $\mathcal{C}_{n}$. Значение же функции $E$ на каждой гиперплоскости меняется со временем и представляет волну с опрокидывающимся фронтом. Кривая $\mathcal{C}_{n}$ фактически представляет собой проекцию характеристики ривертона на вещественное координатное пространство $\mathbb{R}^{n}$. Сама характеристика является траекторией фронта волны в пространстве-времени $\mathbb{R}^{n} \times T$, где $T$ - ось времени.

Заметим, что для $n=1$ кривая $\mathcal{C}_{n}$ совпадает с одномерной осью $x^{1}$, а уравнение (1) переходит в уравнение Хопфа (10).

В случае, когда кривая $\mathcal{C}_{n}$ не является прямой, гиперплоскости, ортогональные кривой $\mathcal{C}_{n}$, будут пересекаться по некоторым гиперплоскостям. Совокупность всех точек пересечения гиперплоскостей, ортогональных $\mathcal{C}_{n}$, заполняет в общем случае некоторую область $\mathcal{V}^{n} \subset \mathbb{R}^{n}$, которая целиком определяется геометрией кривой $\mathcal{C}_{n}$. В общем случае в области $\mathcal{V}^{n}$ лежат точки, на которых гиперплоскости $s=\mathrm{const}$ пересекаются бесконечное число раз. На границе этой области число пересечений конечно. Поэтому в области $\mathcal{V}^{n}$ непрерывные решения отсутствуют. В некотором смысле границы областей $\mathcal{V}^{n}$ подобны каустикам в оптике, но определяются с геометрической точки зрения иначе.

Отметим также то обстоятельство, что ривертоны по сути являются решениями задачи Коши для уравнений (1) и (4). Этот момент важен, поскольку в утверждении 4 кривая $\mathcal{C}_{n}$ задается в начальный момент времени вместе с начальным 
распределением функции $E_{0}=E(s, 0)$ вдоль этой кривой. Это означает, что вместе с геометрическими характеристиками кривой $\mathcal{C}_{n}$ задаются пространственные свойства ривертона, которые определяются геометрией областей однозначности функции $E(\mathbf{x}, t)$.

\section{5. ОБЩАЯ ГЕОМЕТРИЧЕСКАЯ СТРУКТУРА РИВЕРТОНОВ}

Пусть задана некоторая базовая кривая $\mathcal{C}_{n}$ ривертона в форме системы уравнений (9). Интегрируя эту систему, приходим к параметрическому заданию данной кривой в форме уравнений

$$
x^{\alpha}=X^{\alpha}(s)=\int n^{\alpha}(s) d s+x_{0}^{\alpha} .
$$

Постоянные $x_{0}^{\alpha}$ указывают положение кривой относительно начала координат. В этом случае гиперплоскости уровня $E=$ const в пространстве $\mathbb{R}^{n}$, соответствующие заданному значению параметра $s$, можно задать уравнением

$$
\sum_{\alpha=1}^{n} x^{\alpha} n^{\alpha}(s)=\sum_{\alpha=1}^{n} X^{\alpha}(s) n^{\alpha}(s)=\frac{1}{2} \frac{d}{d s} \sum_{\alpha=1}^{n}\left[X^{\alpha}(s)\right]^{2} .
$$

Две гиперплоскости, отвечающие двум значениям $s_{1}$ и $s_{2}$ параметра $s$, пересекаются в пространстве $\mathbb{R}^{n}$ по совокупности точек, являющихся решением двух таких уравнений:

$$
\sum_{\alpha=1}^{n} x^{\alpha} n^{\alpha}\left(s_{1}\right)=F\left(s_{1}\right), \quad \sum_{\alpha=1}^{n} x^{\alpha} n^{\alpha}\left(s_{2}\right)=F\left(s_{2}\right),
$$

где

$$
F(s)=\frac{1}{2} \frac{d}{d s} \sum_{\alpha=1}^{n}\left[X^{\alpha}(s)\right]^{2} .
$$

Эти совокупности точек образуют в $\mathbb{R}^{n}$ гиперплоскости размерности $n-2$. Огибающая множества всех таких гиперплоскостей будет образовывать границу областей однозначности и многозначности ривертона.

Огибающую области многозначности без ограничения общности можно вычислить следующим образом. Она состоит из точек пересечения всех гиперплоскостей, бесконечно близких при $s_{1} \rightarrow s_{2}$. Систему (13) можно разрешить относительно первых двух координат $x_{1}$ и $x_{2}$, в результате чего они становятся функциями $s_{1}$ и $s_{2}$, а также остальных координат $x_{3}, \ldots, x_{n}$ :

$$
\begin{aligned}
& x_{1}\left(s_{1}, s_{2}\right)=\frac{G\left(s_{2}, \mathbf{x}\right) n^{2}\left(s_{1}\right)-G\left(s_{1}, \mathbf{x}\right) n^{2}\left(s_{2}\right)}{n^{2}\left(s_{1}\right) n^{1}\left(s_{2}\right)-n^{2}\left(s_{2}\right) n^{1}\left(s_{1}\right)}, \\
& x_{2}\left(s_{1}, s_{2}\right)=-\frac{G\left(s_{2}, \mathbf{x}\right) n^{1}\left(s_{1}\right)-G\left(s_{1}, \mathbf{x}\right) n^{1}\left(s_{2}\right)}{n^{2}\left(s_{1}\right) n^{1}\left(s_{2}\right)-n^{2}\left(s_{2}\right) n^{1}\left(s_{1}\right)},
\end{aligned}
$$

где

$$
G(s, \mathbf{x})=F(s)-\sum_{\alpha=3}^{n} x^{\alpha} n^{\alpha}(s) .
$$

В пределе $s_{1} \rightarrow s_{2} \rightarrow s$ мы получаем уравнение гиперплоскости $\mathcal{E}^{n-2}\left(s_{1}, s_{2}\right)$ размерности $n-2$, являющейся пересечением двух бесконечно близких гиперплоскостей 


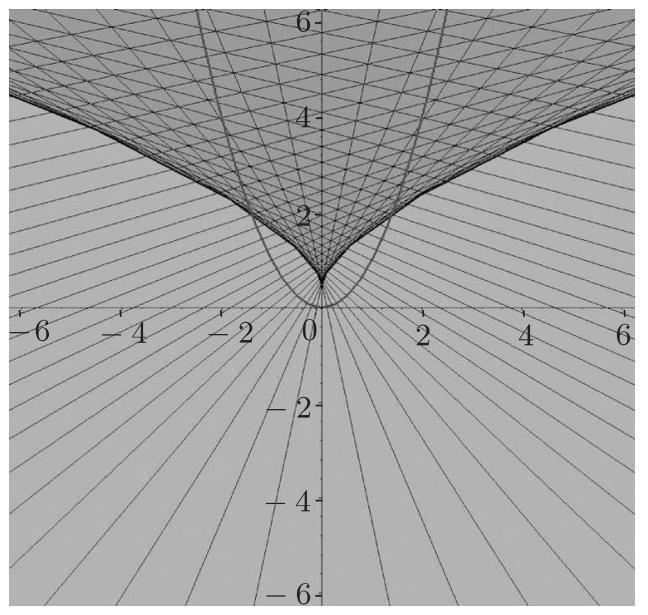

a

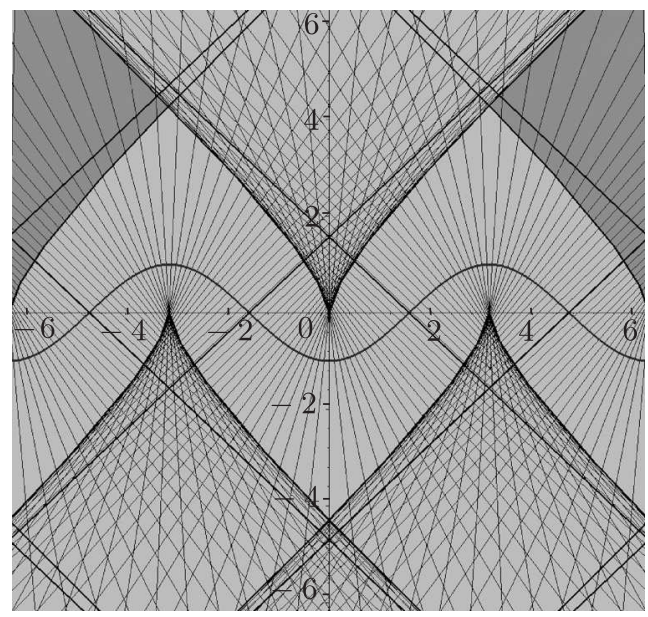

6

Рис. 1. Области однозначности ривертонов (выделены светлым серым) для базовых кривых $y=x^{2}$ (а) и $y=-\cos x$ (б) (сплошная кривая).

$E=E\left(s_{1}\right)$ и $E=E\left(s_{2}\right)$. В пределе $s_{1} \rightarrow s_{2} \rightarrow s_{0}$ получаем множество $\left\{\mathcal{E}^{n-2}\left(s_{0}\right)\right\}$ гиперплоскостей, зависящих от параметра $s_{0}$, образующих гиперповерхность $\mathcal{G}^{n-1}$, к которой касательны все гиперплоскости $E=E\left(s_{0}\right)$ для значений параметра $s_{0}$ в некотором интервале $\left(s_{1}, s_{2}\right)$. Поскольку все гиперплоскости $E=E(s)$ из интервала $s \in\left(s_{1}, s_{2}\right)$ касательны к $\mathcal{G}^{n-1}$, они пересекаются друг с другом в области, лежащей в $\mathbb{R}^{n}$ с одной стороны по отношению к $\mathcal{G}^{n-1}$. Следовательно, гиперповерхность $\mathcal{G}^{n-1}$ разделяет области многозначности и однозначности ривертона, по крайней мере, для интервала $\left(s_{1}, s_{2}\right)$ параметра $s$.

Переходя к пределу $s_{1} \rightarrow s_{2} \rightarrow s$ в соотношениях (14), получаем уравнения параметрического задания гиперповерхностей $\mathcal{G}^{n-1}$ :

$$
x_{1}(s)=\frac{G_{1}^{\prime}(s, \mathbf{x})}{N_{1}^{\prime}(s)}, \quad x_{2}(s)=\frac{G_{2}^{\prime}(s, \mathbf{x})}{N_{2}^{\prime}(s)},
$$

где

$$
\begin{aligned}
G_{1}^{\prime}(s, \mathbf{x}) & =\frac{d}{d s}\left(\frac{G(s, \mathbf{x})}{n^{1}(s)}\right), & G_{2}^{\prime}(s, \mathbf{x}) & =\frac{d}{d s}\left(\frac{G(s, \mathbf{x})}{n^{2}(s)}\right), \\
N_{1}^{\prime}(s) & =\frac{d}{d s}\left(\frac{n^{1}(s)}{n^{2}(s)}\right), & N_{2}^{\prime}(s) & =\frac{d}{d s}\left(\frac{n^{2}(s)}{n^{1}(s)}\right) .
\end{aligned}
$$

На рис. 1 приведены примеры вычисления областей однозначности ривертонов для размерности координатного пространства $n=2$ в случае кривых $y=x^{2}$ (рис. 1a) и $y=-\cos x$ (рис. 1б). На рисунках сплошными прямыми обозначены линии уровня $E=$ const.

Границы областей многозначности (на рисунках обозначены жирной кривой) задаются параметрически следующими соотношениями:

$$
X(s)=-4 s^{3}, \quad Y(s)=\frac{1+6 s^{3}}{2} ;
$$




$$
X(s)=s-2 \operatorname{tg} s+\frac{\sin 2 s}{2}, \quad Y(s)=\frac{1-\cos 2 s}{\cos s}
$$

для рис. 1а и 16 соответственно.

Рассматривая общие свойства огибающих, которые задаются уравнениями (15), легко понять, что в случае замкнутых кривых или кривых с самопересечениями области однозначности решений в форме ривертона отсутствуют. Однако в этом случае, дополняя решение некоторыми условиями периодичности, можно, по крайней мере, для некоторых кривых, например окружностей, построить однозначные решения. Мы не будем доказывать этот факт специально, поскольку для окружностей он практически очевиден, а для более общего случая требует детального анализа, что выходит за рамки настоящей работы.

\section{6. ОПРОКИДЫВАНИЕ ФРОНТА}

Области неоднозначности $\mathcal{V}^{n}$ - не единственная особенность ривертонов. Некоторые изоповерхности функции $E(\mathbf{x}, t)$ в пространстве $\mathbb{R}^{n}$ являются целиком поверхностями опрокидывания фронта волны в соответствии со свойствами уравнения Хопфа (10). Все точки пространства, в которых происходит ветвление решения или опрокидывание его фронта, определяются из условия обращения производных решения по координатам в бесконечность. По аналогии с этим мы можем найти значения параметра $s$, при которых происходит опрокидывание фронта ривертонов. Из (3) имеем

$$
E_{, \alpha}=-\frac{A_{\alpha} H_{\eta}}{H_{\xi}+\left(\mathbf{A}^{\prime}, \mathbf{x}\right) H_{\eta}} .
$$

Отсюда находим общее уравнение для поверхности опрокидывания фронта:

$$
H_{\xi}+\left(\mathbf{A}^{\prime}, \mathbf{x}\right) H_{\eta}=0, \quad H(\xi, \eta)=0 .
$$

В области однозначности поверхность опрокидывания фронта представляет собой некоторую гиперплоскость $E=E\left(s_{*}\right)$ со значением параметра $s=s_{*}$, которое находится из аналогичного соотношения для уравнения Хопфа (10):

$$
s_{*}=-\frac{H_{\xi}}{R^{\prime}\left(E\left(s_{*}, t_{*}\right)\right)},
$$

где $H(\xi, \eta)$ - та же функция, что и в решении уравнения (2).

Для полноты анализа укажем на следующее свойство ривертонов.

УтвЕРЖДЕНИЕ 5. Пусть $E(\mathbf{x}, t)$ - решение системъ (1) в виде ривертона. Тогда любая функиия $\widetilde{E}(\mathbf{x}, t)=F(E(\mathbf{x}, t))$, где $F(E)$ - произвольная дважды дифференцируемая функиия одного аргумента $E$, также является ривертоном.

ДоказАтельство. Пусть $E(\mathbf{x}, t)$ - ривертон, а $F(E)$ - произвольная дважды дифференцируемая функция. Тогда имеем следующие тождества:

$$
\frac{\partial F(E)}{\partial x^{\alpha}}=F^{\prime}(E) E_{, \alpha}=F^{\prime}(E) A_{\alpha} E_{t}=A_{\alpha} \frac{\partial F(E)}{\partial t} .
$$

Эти тождества соответствуют обращению в тождество уравнений (1) и, следовательно, уравнения (4) при подстановке в них в качестве решения $\widetilde{E}(\mathbf{x}, t)=F(E(\mathbf{x}, t))$. 


\section{7. МНОГОЗНАЧНЫЕ РЕШЕНИЯ УРАВНЕНИЙ ДАЛАМБЕРА}

Среди уравнений (4), решениями которых являются ривертоны, полезно выделить особый класс, соответствующий условию $R(E)=|\mathbf{A}(E)|=1$. При выполнении этого условия уравнение (4) представляет собой уравнение Даламбера в вещественном координатном пространстве $\mathbb{R}^{n}$. В результате можно доказать, что при любом $n>1$ линейное уравнение Даламбера имеет решения в виде ривертонов (2):

$$
H\left(E, t+\sum_{\alpha=1}^{n} n_{\alpha}(E) x^{\alpha}\right)=0
$$

т. е. многозначные решения. Для случая $n=3$ подобный результат был использован в теории твисторов (см., например, [7], [8]), но для комплексных функций $E$. В данном случае решения вещественные, если входящие в выражение (17) произвольные функции являются вещественными.

Таким образом, можно сформулировать

УтвеРЖДЕНИЕ 6. Для любого $n>1$ уравнение Даламбера

$$
\Delta E=E_{, t t}, \quad \diamond_{1} E=0,
$$

имеет решения $E(\mathbf{x}, t)$ в виде ривертонов $\left\{\mathcal{C}_{n}, E(s, t)\right\}$, структура которых определяется произвольной вектор-функиией $\mathbf{n}(E),|\mathbf{n}|^{2}=1$, и интегралом движения $H(\xi, \eta)$.

Поскольку уравнение Даламбера линейное, то для него выполняется линейный принцип суперпозиции. Это означает, что любая линейная комбинация ривертонов является решением уравнения Даламбера. Однако при этом следует иметь в виду, что каждый ривертон имеет свои области неоднозначности $\mathcal{V}^{n}\left[\mathcal{C}_{n}\right]$. В суперпозиционном решении область неоднозначности будет являться объединением областей неоднозначности отдельных ривертонов. Следует также отметить, что наличие гиперповерхностей, на которых решение типа ривертона становится неоднозначным, эквивалентно наличию сингулярных источников в правой части уравнения Даламбера. Аналогичные конструкции рассматривались в работах [8]. Подобные построения, по всей видимости, имеют место и в случае нелинейных уравнений (4) общего вида, но это требует отдельного анализа.

\section{8. ТОЧНЫЕ РЕШЕНИЯ МНОГОМЕРНЫХ НЕЛИНЕЙНЫХ ТЕЛЕГРАФНЫХ УРАВНЕНИЙ И УРАВНЕНИЯ КЛЕЙНА-ГОРДОНА}

Ривертоны позволяют строить точные решения других многомерных нелинейных уравнений. Доказательство такого общего утверждения строится на простом анализе суперпозиций ривертонов и некоторых элементарных функций.

Рассмотрим функции следующего вида:

$$
\Phi(\mathbf{x}, t)=G(E+L(\mathbf{k}, \omega, \mathbf{x}, t)),
$$

где $E-$ ривертон, $L(\mathbf{k}, \omega, \mathbf{x}, t)=(\mathbf{k}, \mathbf{x})+\omega t$ есть линейная функция координат и вре- 
мени, $G(S)$ есть дважды дифференцируемая функция одного аргумента $S(E, \mathbf{x}, t)=$ $E+(\mathbf{k}, \mathbf{x})+\omega t, \mathbf{k}=\left(k_{1}, \ldots, k_{n}\right)$ - постоянный вектор в пространстве $\mathbb{R}^{n}, \omega$ - вещественное число. Имеем следующее общее тождество:

$$
\diamond_{|\mathbf{A}|} \Phi=\diamond_{|\mathbf{A}|} G(S)=G^{\prime}(S) \diamond_{|\mathbf{A}|} S+G^{\prime \prime}(S)\left[(\nabla S, \nabla S)-|\mathbf{A}|^{2}\left(S_{t}\right)^{2}\right] .
$$

Учитывая свойства ривертонов и линейность функции $L(\mathbf{k}, \omega, \mathbf{x}, t)$, имеем

$$
\diamond_{|\mathbf{A}|} S(E, \mathbf{x}, t)=0, \quad L_{t t}=0 .
$$

Кроме того, находим

$$
\begin{aligned}
(\nabla S, \nabla S) & =2(\mathbf{k}, \nabla E)+|\mathbf{k}|^{2}-|\mathbf{A}(E)|^{2}\left(\omega^{2}+2 \omega E_{t}\right)= \\
& =2\left[(\mathbf{k}, \mathbf{A}(E))-\omega|\mathbf{A}(E)|^{2}\right] E_{t}+|\mathbf{k}|^{2}-|\mathbf{A}(E)|^{2} \omega^{2}
\end{aligned}
$$

Отсюда вытекает

УтВеРЖДЕНИЕ 7. Функиия $G(S)$, где $S=E+L(\mathbf{k}, \omega, \mathbf{x}, t), E-$ ривертон, $a$ $L(\mathbf{k}, \omega, \mathbf{x}, t)=(\mathbf{k}, \mathbf{x})+\omega t-$ линейная функция координат и времени с произвольными вещественными коэффициентами $\mathbf{k}, \omega$, при условиях

$$
|\mathbf{A}|^{2}=R_{0}^{2}=\text { const }, \quad(\mathbf{k}, \mathbf{A}(E))-R_{0}^{2} \omega=\lambda=\text { const }
$$

является решением нелинейного телеграфного уравнения

$$
\diamond_{R_{0}} G(S)=G^{\prime \prime}(S)\left(2 \lambda S_{t}+\mu\right)
$$

где постоянная $\mu$ имеет следующий вид:

$$
\mu=|\mathbf{k}|^{2}-\omega^{2} R_{0}^{2}-2 \omega \lambda .
$$

Решения этого типа будем называть аддитивно модифицированными ривертонами.

ПримеР 1. В случае $\lambda=0$ уравнение (19) принимает вид нелинейного уравнения Клейна-Гордона

$$
\left(\Delta-\frac{\partial^{2}}{\partial t^{2}}\right) G(S)=\mu G^{\prime \prime}(S) .
$$

а в частном, когда $G(S)=\ln S$ получаем уравнение Лиувилля

$$
\left(\Delta-\frac{\partial^{2}}{\partial t^{2}}\right) \ln S=-\frac{\mu}{S^{2}},
$$

а в случае, когда функция $G(S)$ удовлетворяет уравнению $G^{\prime \prime}=\nu^{2} G$, приходим к линейному уравнению Клейна-Гордона

$$
\left(\Delta-\frac{\partial^{2}}{\partial t^{2}}\right) G(S)=\mu \nu^{2} G(S) .
$$

Это указывает на существование решений в форме аддитивно модифицированного ривертона для этого типа уравнений. 
ПримеР 2. В случае $\mu=0$ уравнение (19) принимает вид нелинейного телеграфного уравнения

$$
\left(\Delta-\frac{\partial^{2}}{\partial t^{2}}\right) G(S)=\lambda G^{\prime \prime}(S) S_{t} .
$$

В случаях $n=2,3$ это уравнение имеет отношение к распространению нелинейных электромагнитных волн в среде с нелинейной проводимостью, например в газах.

Прямым следствием утверждений 5 и 7 является следующее утверждение.

УтвеРЖДЕНИЕ 8. Функиия $G(T)$, где $T=e^{L(\mathbf{k}, \omega, \mathbf{x}, t)} E, E-$ ривертон, а функиия $L(\mathbf{k}, \omega, \mathbf{x}, t)=(\mathbf{k}, \mathbf{x})+\omega t$, при выполнении тех же условий (18) и (20) удовлетворяет уравнению

$$
\diamond_{R_{0}} G(T)=\left(G^{\prime}(T)+G^{\prime \prime}(T) T\right)\left[(\mu-\lambda \omega) T+\lambda T_{t}\right] .
$$

Этот тип решений назовем мультипликативно модифицированным ривертоном.

\section{9. ЗАКЛЮЧЕНИЕ}

В работе построен новый класс точных решений нелинейных гиперболических уравнений второго порядка с вещественными коэффициентами в случае произвольной размерности координатного пространства. Новый класс решений - ривертоны строится как вещественное решение задачи Коши системы квазилинейных уравнений первого порядка. Ривертоны представляют собой плоские волны с произвольно заданной траекторией направления распространения фронта волны. Универсальность построенного класса решений состоит в том, что он существует для очень широкого класса нелинейных гиперболических уравнений второго порядка, включающего целый ряд важных с точки зрения приложений уравнений математической физики. К ним, в частности, относятся уравнения распространения электромагнитных волн в среде с нелинейной поляризацией и проводимостью, нелинейных акустических волн, а также гидродинамические уравнения. При сравнительно простой форме описания ривертонов их структура оказывается достаточно сложной и содержит два основных элемента. Первый - это опрокидывающаяся волна с плоским фронтом, а второй элемент - статическое, неизменное во времени расположение областей однозначности и многозначности решений. В работе приведено общее решение проблемы расположения в пространстве областей однозначности и многозначности с помощью вычисления огибающей множества точек пересечения бесконечно близких гиперплоскостей уровня. Вместе с тем в работе не рассматривался вопрос о том, какие граничные условия выделяют ривертоны среди всех других возможных решений уравнений, допускающих такие решения. Это, в частности, относится и к уравнениям типа уравнения Даламбера. Данный вопрос имеет важное значение для прикладных задач, но требует отдельного анализа, который выходит за рамки настоящей работы.

Наиболее существенным свойством ривертонов является их относительная универсальность как по отношению к размерности координатного пространства, так и по отношению к типу уравнений. Как показано в нашей работе, при небольших модификациях ривертонов они становятся решениями большого класса нелинейных уравнений типа уравнения Клейна-Гордона в размерности $n+1$ и нелинейных 
телеграфных уравнений. Это указывает на то, что явления, связанные с опрокидыванием волн и их многозначностью, являются достаточно распространенными среди уравнений гиперболического типа и присущи даже линейным уравнениям этого типа.

Благодарности. Настоящая работа выполнена в рамках федеральных целевых программ "Исследования и разработки по приоритетным направлениям развития научно-технологического комплекса России на 2007-2012 г." и "Научные и научнопедагогические кадры инновационной России на 2009-2013 г.", а также в рамках государственного задания Министерства образования и науки России № 2.1894.2011 и при частичной поддержке РФФИ (грант № 11-01-007_а).

\section{Список литературы}

[1] Б. Л. Рождественский, Н. Н. Яненко, Системы квазилинейных уравнений и их приложения к газовой динамике, Наука, М., 1978.

[2] А. Г. Куликовский, Е. И. Свешникова, А. П. Чугайнова, "Математические методы изучения разрывных решений нелинейных гиперболических систем уравнений”, Лекц. курсы НОЦ, 16, МИАН, М., 2010, 3-120.

[3] Дж. Уизем, Линейные и нелинейные волны, Мир, М., 1977.

[4] О.И. Богоявленский, Опрокидывающиеся солитоны. Нелинейные интегрируемые уравнения, Наука, М., 1991.

[5] И. Г. Катаев, Ударные электромагнитные волны, Советское радио, М., 1963.

[6] А. А. Акопян, Д. Л. Оганесян, Квантовая электроника, 24:7 (1997), 622-624.

[7] R. Penrose, W. Rindler, Spinors and Space-Time, v. 2: Spinor and Twistor Methods in Space-Time Geometry, Cambridge Univ. Press, Cambridge, 1986.

[8] V.V. Kassandrov, "Algebrodynamics: primordial light, particles-caustics and the flow of time", Space-Time Structure: Algebra and Geometry, eds. D. G. Pavlov, Gh. Atanasiu, V. Balan, Lilia Print, Moscow, 2007, 422-440; "Quaternionic analysis and the algebrodynamics", 441-473; arXiv:0710.2895; Phys. Atom. Nucl., 72:5 (2009), 813-827, arXiv: 0907.5425 .

[9] В. М. Журавлев, Известия вузов. Сер. Прикладная нелинейная динамика, 9:6 (2001), $115-128$.

[10] В. М. Журавлев, "Опрокидывающиеся электромагнитные волны в диэлектриках и проводящей среде”, Тезисы докладов XLVIII Всероссийской конференции по проблемам физики частич, физики плазмы и конденсированных сред, оптоэлектроники, посвященной 100-летию профессора Я.П. Терлецкого (Москва, 15-18 мая 2012 г.), РУДН, M., 2012, 137.

[11] С. П. Царев, Изв. АН СССР. Сер. матем., 54:5 (1990), 1048-1068.

[12] E. V. Ferapontov, K. R. Khusnutdinova, Commun. Math. Phys., 248:1 (2004), 187-206, arXiv: nlin/0305044; J. Phys. A, 37:8 (2004), 2949-2963, arXiv: nlin/0310021; J. Math. Phys., 45:6 (2004), 2365-2377.

[13] Е. В. Ферапонтов, К. Р. Хуснутдинова, М. В. Павлов, ТМФ, 144:1 (2005), 35-43.

Поступила в редакцию 11.07.2012, после доработки 8.08.2012 